\title{
Article
}

\section{Effect of Functionalized Graphene Oxide with Hyper-Branched Flame Retardant on Flammability and Thermal Stability of Cross-Linked Polyethylene}

Hu, Weizhao, Zhan, Jing, Wang, Xin, Hong, Ningning, Wang, Bibo, Song, Lei, Stec, Anna A, Hull, T'Richard, Wang, Jian and Hu, Yuan

Available at https://clok.uclan.ac.uk/10863/

Hu, Weizhao, Zhan, Jing, Wang, Xin, Hong, Ningning, Wang, Bibo, Song, Lei, Stec, Anna A orcid iconORCID: 0000-0002-6861-0468, Hull, T Richard orcid iconORCID: 0000-0002-7970-4208, Wang, Jian et al (2014) Effect of Functionalized Graphene Oxide with Hyper-Branched Flame Retardant on Flammability and Thermal Stability of Cross-Linked Polyethylene. Industrial and Engineering Chemistry Research, 53 (8). pp. 3073-3083. ISSN 0888-5885

It is advisable to refer to the publisher's version if you intend to cite from the work. http://dx.doi.org/10.1021/ie4026743

For more information about UCLan's research in this area go to http://www.uclan.ac.uk/researchgroups/ and search for <name of research Group>.

For information about Research generally at UCLan please go to http://www.uclan.ac.uk/research/

All outputs in CLoK are protected by Intellectual Property Rights law, including Copyright law. Copyright, IPR and Moral Rights for the works on this site are retained by the individual authors and/or other copyright owners. Terms and conditions for use of this material are defined in the policies page. 


\title{
Effect of Functionalized Graphene Oxide with Hyper-Branched Flame Retardant on Flammability and Thermal Stability of Cross- Linked Polyethylene
}

\author{
Weizhao Hu, ${ }^{\dagger}$ Jing Zhan, ${ }^{\dagger}$ Xin Wang, ${ }^{\dagger}$ Ningning Hong, ${ }^{\dagger}$ Bibo Wang, ${ }^{\dagger}$ Lei Song, ${ }^{\dagger}$ Anna A Stec, ${ }^{\S}$ \\ T. Richard Hull, ${ }^{\S}$ Jian Wang, ${ }^{*,+, \neq}$ and Yuan $\mathrm{Hu}^{*^{+,}, \neq}$ \\ ${ }^{\dagger}$ State Key Laboratory of Fire Science, University of Science and Technology of China, Anhui 230026, People's Republic \\ of China \\ ${ }^{\ddagger}$ Suzhou Key Laboratory of Urban Public Safety, Suzhou Institute for Advanced Study, University of Science and \\ Technology of \\ China, Suzhou, Jiangsu 215123, People's Republic of China \\ ${ }^{\S}$ Centre for Fire and Hazards Science, University of Central Lancashire, Preston PR1 2HE, United Kingdom
}

\begin{abstract}
In this work, GO was functionalized by a hyper-branched flame retardant, which was synthesized by the reaction of $\mathrm{N}$-aminoethyl piperazine and di(acryloyloxyethyl)methylphosphonate. Subsequently, the resultant functionalized GO (FGO) was incorporated into the cross-linked polyethylene (XLPE) to enhance the flame retardancy of the matrix. Transmission electron spectroscopy images indicated that FGO exhibited uniform dispersion in XLPE matrix and strong adhesion with the matrix by crosslinking, which improved barrier effect due to reduced heat release and the free radical transfer between the matrix and graphene nanosheets. The incorporation of FGO into XLPE matrix endowed polymer composites with flame retardancy and thermal stability. In addition, the homogeneous dispersion of functionalized GO with a hyper-branched flame retardant in the polymer matrix improved the antioxidation and mechanical properties of XLPE-FGO nanocomposites compared to the XLPE- GO samples, as demonstrated through the oxidative induction temperature and time test, oven ageing test and mechanical test.
\end{abstract}

\section{INTRODUCTION}

In the past decade, graphene has gained considerable attention in fabricating polymer nanocomposites since its discovery in $2004^{1}$. Due to its ultrahigh electrical conductivity, thermal stability and mechanical properties, graphene has been extensively used as the nanofiller in polymers to improve the electrical, thermal and mechanical properties of nanocomposites. ${ }^{2-4}$ Various methods have been developed to synthesize graphene, such as mechanical exfoliation, thermal deposition, chemically reduced graphite oxide and liquid-phase exfoliation of graphite. ${ }^{5-8}$

For an industrial application, graphene oxide (GO) and reduced graphene oxide are widely produced from the chemical exfoliation method, which contain the hydroxyl, epoxy and carboxyl groups on the basal planes and edges. ${ }^{9,10}$ However, the reaggregation tendency and the incompatibility with the polymer matrix lead to the poor dispersion of graphene. Functionalization of graphene is essential for improving its dispersion in polymer matrix, especially the nonpolar polymers, such as polyethylene (PE) and polypropylene (PP). ${ }^{11,12}$ Functionalized graphene has been focused on the surface modification by using the organic compounds with the active groups, such as isocyanate, amino, acyl chloride, diazonium, silane coupling agent, etc. ${ }^{13-18}$ 
Recently, graphene or functionalized graphene has attracted increasing interest for its potential application as a flame retardant additive due to its special two-dimensional atomic carbon sheet structure. An interesting work shows that functionalized graphene oxide (FGO) with hexachlorocyclo- triphosphazene (HCTP) enhances the mechanical property, electrical property and thermal stability of epoxy resin. ${ }^{19}$ A further study verifies that functionalized GO with HCTP can obviously increase the char yield and decrease the peak heat release rate (pHRR), CO release rate and $\mathrm{CO}_{2}$ release rate in polystyrene. ${ }^{20}$ Other significant research demonstrates that functionalized GO with low molecular intumescent flame retardant not only improves the dispersion of graphene butalso effectively increases the flame retardancy of ethylene/vinyl acetate nanocomposites. ${ }^{21}$

Hyper-branched polymers have gained considerable attention because of their highly branched and nonentangled architecture as well as a large number of terminal groups, which can facilitate further modification with a target polymer via covalent linkage. ${ }^{22,23}$ Additionally, hyper-branched polymers are prepared by an A2B3 type polymerization that monomer " $A$ " has two functional group and monomer " $\mathrm{B}$ " possesses three functional groups. It is well-known that $\mathrm{N}$-aminoethyl piperazine (AEPZ) is an effective vinyl-reactive agent via Michael addition reaction; meanwhile, it can be conjugated onto GO using amino-coupling reaction. ${ }^{24}$ Typically, hyper- branched polyphosphonate acrylate is synthesized as a flame retardant by employing an A2B3 type polymerization, where di(acryloyloxyethyl) benzenephosphonate (DAMP) containing two vinyl groups is used as an A2 component and AEPZ is used as a B3 component. ${ }^{25}$

Inspired by this idea, functionalized graphene with AEPZ is prepared via surface modification; subsequently, hyper-branched polymer functionalized GO (FGO) is synthesized by in situ polymerization among AEPZ, DAMP and AEPZ modified GO. FGO is blended with the different ratios of polyethylene (PE) to prepare a series of FGO/PE composites. FGO/PE composites are then exposed to irradiation to obtain the cross-linked PE (XLPE). As a comparison, GO/PE composites are also prepared. Functionalization of graphene oxide is expected to not only enhance the dispersion of GO in the XLPE matrix but also covalently link to the XLPE network. A complementary study on the morphology, flammability, thermal stability, thermal ageing properties, and mechanical characteristics of the XLPE composites are investigated and discussed in this article.

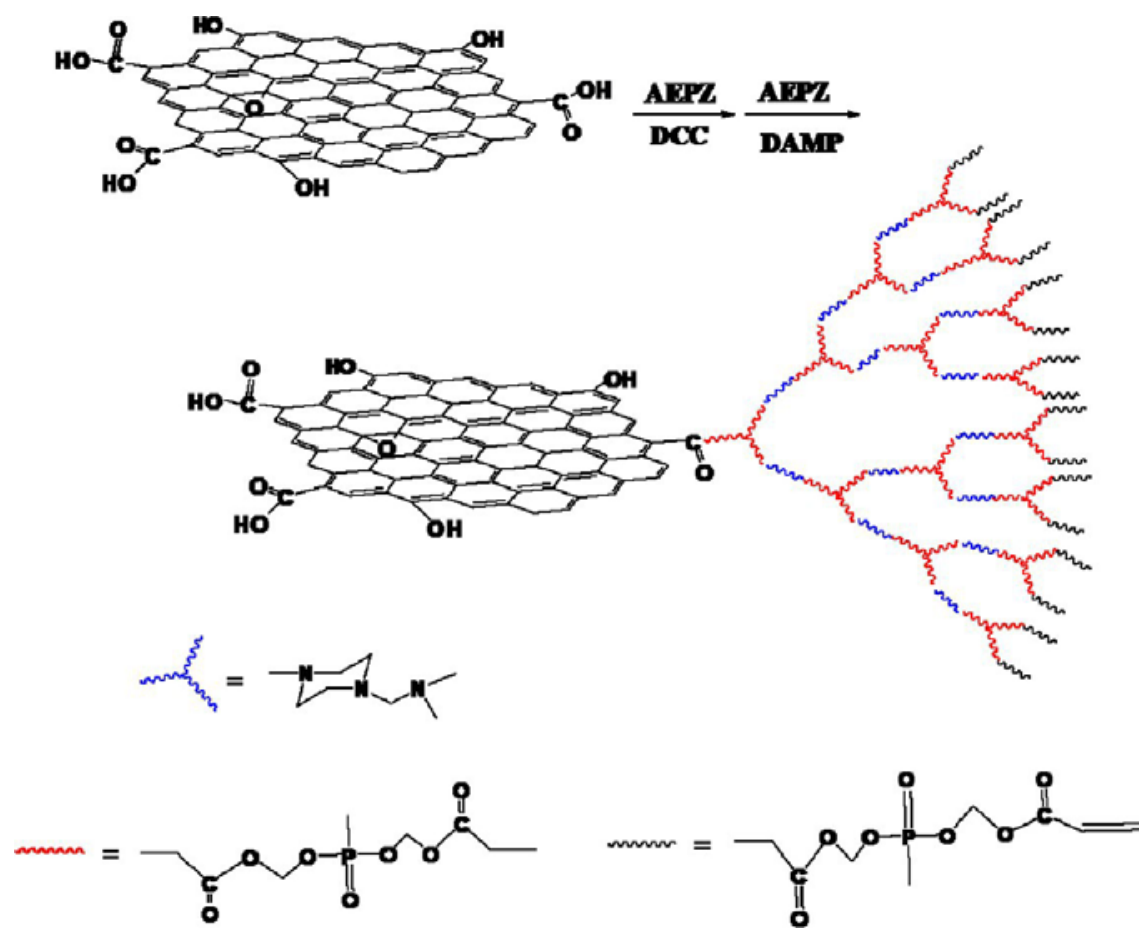

Figure 1. Schematic of the fabrication of functionalized GO. 


\section{EXPERIMENTAL SECTION}

Materials. Natural graphite, potassium permanganate ( $\mathrm{KMnO} 4)$, ammonia (25-28\% aq), hydrazine hydrate $(85 \% \mathrm{aq})$, hydrogen peroxide $\left(\mathrm{H}_{2} \mathrm{O}_{2}, 30 \% \mathrm{aq}\right)$, hydrochloric acid $\left(\mathrm{HCl}, 37 \%\right.$ aq), sulphuric acid (anhydrous $\mathrm{H}_{2} \mathrm{SO}_{4}$, 98\%), $N, N$ - dicyclohexylcarbodiimide (DCC, 99.7\%), triethylamine, tetra-hydrofuran (THF), dichloromethane, methanol and toluene were of analytical reagent grade and purchased from Sinopharm Chemical Reagent Co., Ltd. Hydroxyethyl acrylate (HEA) and $\mathrm{N}$-aminoethyl piperazine (AEPZ) were purchased from Aladdin Co., Ltd. Methyldichlorophosphate (MDCP) was obtained from Suzhou Institute for Advanced Study. The AEPZ, HEA and MDCP were purified by distillation. PE (FB2310) was purchased from Borouge Co., Ltd.

Preparation of Functionalized GO. GO was obtained from the Hummers' method and washed with THF to remove water. $1 \mathrm{~g}$ of GO was added in $200 \mathrm{~mL}$ of THF in a $500 \mathrm{~mL}$ three-necked flask, sonicated for 30 min and then stirred with a mechanical stirrer. $5 \mathrm{~g}$ of AEPZ and $10 \mathrm{mg}$ of DCC were added into the solution as the catalysts, and the solution was refluxed at $50{ }^{\circ} \mathrm{C}$ for $12 \mathrm{~h}$. Afterwards, the obtained AEPZ-GO (yield, about $85 \mathrm{wt} \%$ ) was filtered and washed with methanol (about $50 \mathrm{~mL}$ ) and deionized water (about $100 \mathrm{~mL}$ ), and dried under vacuum at $50{ }^{\circ} \mathrm{C}$ for $12 \mathrm{~h}$.

$13.2 \mathrm{~g}$ of MDCP and $25 \mathrm{~g}$ of triethylamine dissolved in $200 \mathrm{~mL}$ of dried THF were introduced into a three-neck flask equipped with a nitrogen inlet, a mechanical stirrer, and the solution was slowly heated to $60{ }^{\circ} \mathrm{C} .32 .2 \mathrm{~g}$ of HEA was dispersed in $40 \mathrm{~mL}$ of THF and then dripped into the mixture over a period of $1 \mathrm{~h}$. The mixture was stirred at $60^{\circ} \mathrm{C}$ for $6 \mathrm{~h}$, keeping in a nitrogen atmosphere. The precipitant was separated after cooling down to room temperature, and then the solvent and the unreacted reactants were removed by extraction and distillation under the reduced pressure. A colorless liquid product was obtained and named as DAMP (yield, 80 wt \%).

The IR spectrum (KBr) of DAMP exhibited absorption at 1186, $1292 \mathrm{~cm}^{-1}(\mathrm{P}=0), 1031 \mathrm{~cm}^{-1}(\mathrm{P}-\mathrm{O}-\mathrm{C}), 1428 \mathrm{~cm}-1$ $(P-C), 1640 \mathrm{~cm}^{-1}(C=C), 1730 \mathrm{~cm}^{-1}(C=O), 2930 \mathrm{~cm}^{-1}\left(-\mathrm{CH}_{2}-\right)$. The shift and splitting pattern of ${ }^{1} \mathrm{H} N M R$ spectrum $\left(\mathrm{CDCl}_{3}\right)$ is $1.55 \mathrm{ppm}(\mathrm{d}, 3 \mathrm{H}), 4.1-4.5 \mathrm{ppm}(\mathrm{m}, 8 \mathrm{H}), 5.90 \mathrm{ppm}(\mathrm{d}, 2 \mathrm{H}), 6.20 \mathrm{ppm}(\mathrm{t}, 2 \mathrm{H}), 6.45 \mathrm{ppm}(\mathrm{d}, 2 \mathrm{H})$.

$1 \mathrm{~g}$ of AEPZ-GO was added in $200 \mathrm{~mL}$ of THF in a $500 \mathrm{~mL}$ three-necked flask sonicated for $30 \mathrm{~min}$. $11.68 \mathrm{~g}$ of DAMP and $2.58 \mathrm{~g}$ of AEPZ-GO were added into the solution via the Michael addition reaction to form the FGO. After the reaction, the suspension was filtered and washed with DMF (about $50 \mathrm{~mL}$ ) and methanol (about $150 \mathrm{~mL}$ ), and then dried under vacuum at $50{ }^{\circ} \mathrm{C}$ for $12 \mathrm{~h}$. The synthesis route of FGO (yield, about $115 \mathrm{wt} \%$ ) is illustrated in Figure 1.

Preparation of XLPE/GO and XLPE/FGO Nanocomposites. PE/GO and PE/FGO masterbatch was prepared by the solvent blending. Two grams of GO or FGO was dispersed into $400 \mathrm{~mL}$ of toluene sonicated for $0.5 \mathrm{~h}$. Then $20 \mathrm{~g}$ of PE was added into the suspension by the strong mechanical stirring at $100^{\circ} \mathrm{C}$. After the solution was stirred for $2 \mathrm{~h}$, the viscous black slurry was dried at $80^{\circ} \mathrm{C}$ for $12 \mathrm{~h}$ in a vacuum oven.
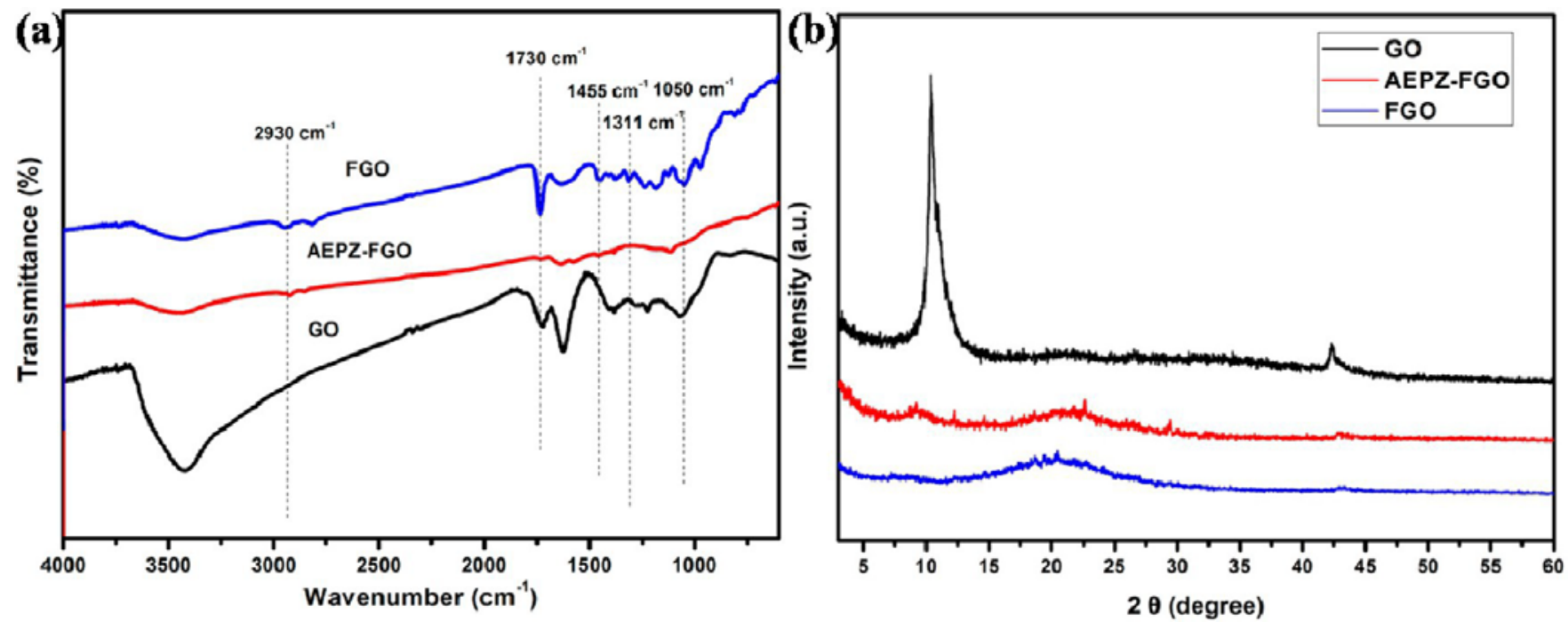

Figure 2. FTIR (a) and XRD (b) spectrum of GO and functionalized GO.

$\mathrm{XLPE} / \mathrm{GO}$ and XLPE/FGO composites were prepared by melt blending at $160{ }^{\circ} \mathrm{C}$ using the different ratios of the crosslinked agent, masterbatch and $\mathrm{PE}$, keeping a roller speed of $60 \mathrm{rpm}$ and mixing for $8 \mathrm{~min}$. The products were formed by hot- pressing at $165{ }^{\circ} \mathrm{C}$ and cutting to fixed dimensions for tests. The prepared samples were irradiated in air condition at room temperature $\left(25 \pm 2{ }^{\circ} \mathrm{C}\right)$ using an electron beam accelerator under forced air cooling. The irradiation dose was selected as $140 \mathrm{kGy}$. 
Characterization. Fourier Transform Infrared Spectroscopy (FTIR). FTIR spectroscopy was performed on a Nicolet $6700 \mathrm{FT}-\mathrm{IR}$ spectrophotometer to characterize the GO and FGO using thin $\mathrm{KBr}$ disc. The transition mode was used and the scanning range of wave number was from 4000 to $500 \mathrm{~cm}^{-1}$.

X-ray Photoelectron Spectroscopy (XPS). The XPS was measured by using an ESCALAB MK II (VG Co., Ltd., England) spectrometer, with Al $\mathrm{K} \alpha$ excitation radiation $(\mathrm{hv}=1253.6 \mathrm{eV})$ in ultrahigh vacuum conditions.

$X$-ray Diffraction (XRD). The XRD were carried out using an X-ray diffractometer (Rigaku Dmax/rA, Japan), using Cu K $\alpha$ radiation $\left(k=0.15418 \mathrm{~nm}\right.$ ) at $40 \mathrm{kV}$ and $20 \mathrm{~mA}$. The scan rate was $4^{\circ} \mathrm{min}^{-1}$. The $\mathrm{GO}$ and FGO were performed as powder and the nanocomposites were performed as thin films (thickness: $1.2 \mathrm{~mm}$ ).

Transmission Electron Microscopy (TEM). The morphology of GO, FGO and XLPE nanocomposites was investigated by a JEM-2100Ftransmission electron microscopy (Electron Optics Laboratory Co., Ltd., Japan). The samples of GO and FGO were dispersed in ethanol with ultrasonication $(0.5 \mathrm{~h})$ and then dripped onto copper grids for observation. The XLPE nanocomposites were room temperature microtomed to ultrathin sections with diamond knife by Du Pont MT-6000 Ultratome.

Scanning Electron Microscopy (SEM). The morphology of the samples was studied by a PHILIPS XL30E scanning electron microscope. The GO and FGO were dispersed in ethanol with ultrasonication ( $0.5 \mathrm{~h})$ and then dripped onto copper sheet, and the XLPE nanocomposites were cryogenically fractured in liquid nitrogen first. Then all the samples were sputter-coated with a conductive layer for observation. The accelerated voltage was $20 \mathrm{KV}$.

Thermogravimetric Analysis (TGA). TGA was carried out using a Q5000 IR thermogravimetric analyzer (TA Instruments) at a linear heating rate of $20{ }^{\circ} \mathrm{C} \mathrm{min}^{-1}$ in nitrogen atmosphere. The weights of all the samples were kept within 5-10 mg. Samples in an open Pt pan were examined under an airflow rate of $60 \mathrm{~mL} \mathrm{~min}^{-1}$ at a temperature ranging from room temperature to $700{ }^{\circ} \mathrm{C}$.

Gel Content. The gel content was tested by a Soxhlet extraction system to extract the cross-linked sample with a solvent of xylene for $48 \mathrm{~h}$. After the solution was dried, the gel content was calculated as the ratio of the weight of insoluble residue and initial sample. Each sample was repeated three times to determine the average gel content.

Cone Calorimeter and Limited Oxygen Index (LOI). The flammability test was performed on the cone calorimeter (FTT, UK) test according to ISO 5660 standard procedures, with the sample dimensions of $100 \times 100 \times 3 \mathrm{~mm}^{3}$. Each specimen was wrapped in aluminum foil and exposed horizontally to $35 \mathrm{~kW} / \mathrm{m}^{2}$ external heat flux. LOI was measured according to ASTMD 2863. The apparatus used was an HC-2 oxygen index meter (Jiangning Analysis Instrument Co., China). The specimens used for the test were of dimensions $100 \times 6.5 \times 3 \mathrm{~mm}$.

Oxidative Induction Temperature (OITm) and Oxidative Induction Time (OITs). The OITm and OITs measurement were evaluated according to the ISO 11357-6-2008. Approximately $10 \mathrm{mg}$ of sample on OITm test was heated in differential scanning calorimeter from 20 to $300^{\circ} \mathrm{C}$ at a rate of $10^{\circ} \mathrm{C} / \mathrm{min}$ with an oxygen flow of $50 \mathrm{~mL} / \mathrm{min}$.

Approximately $10 \mathrm{mg}$ of sample on OITs test was held at $25^{\circ} \mathrm{C}$ for 5 min under a nitrogen flow of $50 \mathrm{~mL} / \mathrm{min}$, and then heated to $220^{\circ} \mathrm{C}$ at a rate of $20^{\circ} \mathrm{C} / \mathrm{min}$ and held at $5 \mathrm{~min}$ for equilibration. Subsequently, the gas was switched to oxygen with the flow rate of $50 \mathrm{~mL} / \mathrm{min}$ and kept the temperature and flow rate for equilibration. The data of OITm and OITs were recorded by the software of Perkin-Elmer differential scanning calorimeter 7 . Each specimen was repeated for three times, and the results indicated good repeatability.

Mechanical Properties. The mechanical properties were measured with a universal testing machine (Instron 1185) with the stretching rate of $100 \mathrm{~mm} / \mathrm{min}$. The shape of the samples was prepared according to the ASTM D412. Each specimen was repeated five times. 

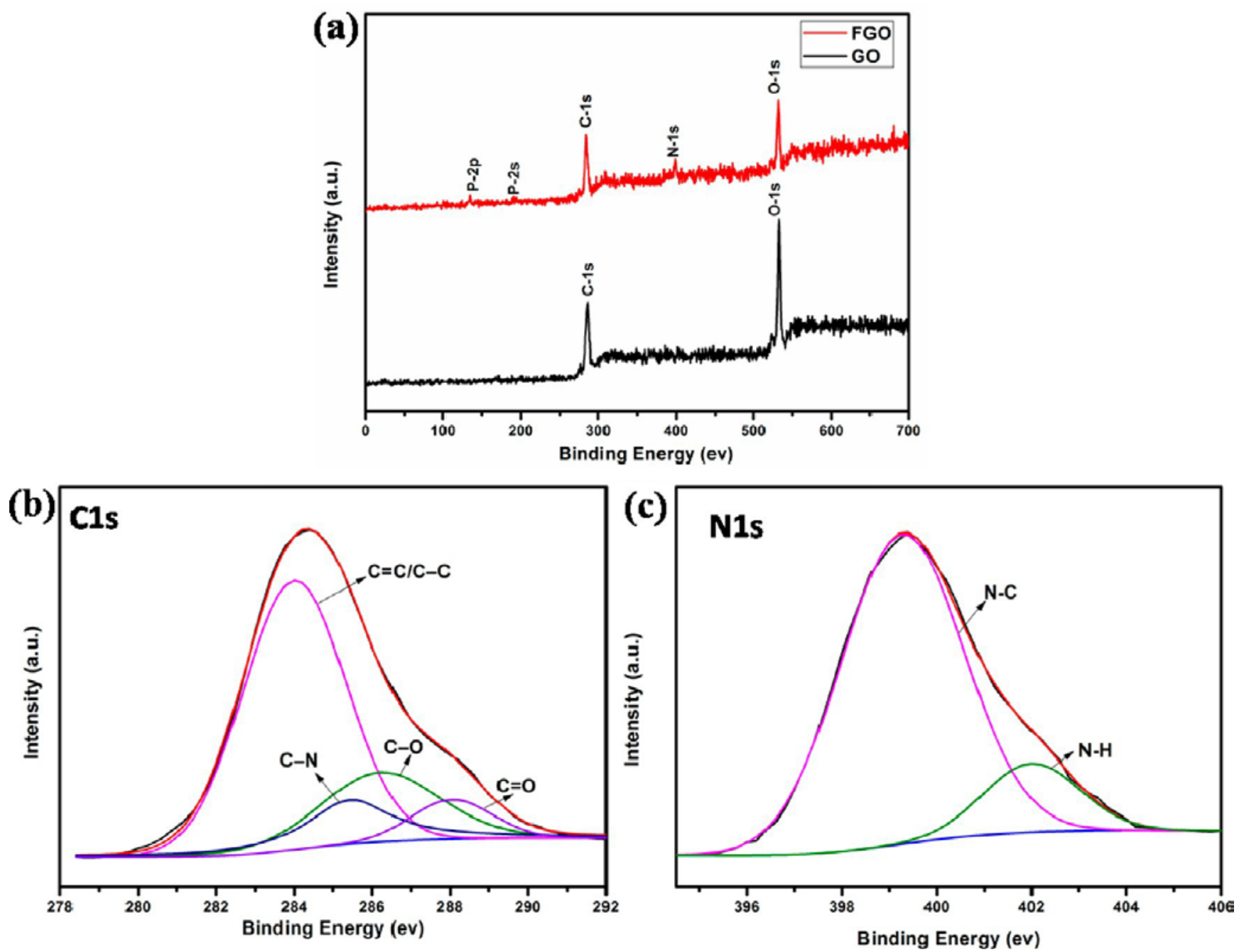

Figure 3. XPS scan of GO and FGO (a), the C1s of FGO (b) and N1s of FGO (c).

\section{RESULTS AND DISCUSSION}

Structural and Morphological Characterization of GO and FGO. The covalent linkage of the hyper-branched flame retardant grafted onto GO is confirmed by FTIR, as shown in

Figure $2 \mathrm{a}$. In the FTIR spectrum of $\mathrm{GO}$, the peak at $1730 \mathrm{~cm}^{-1}$ is assigned to the $\mathrm{C}=\mathrm{O}$ stretching vibration, and the other characteristic absorption bands at 1620,1065 , and $1220 \mathrm{~cm}^{-1}$ are assigned to the stretching vibration of $\mathrm{sp}^{2}$ hybridized carbon, $\mathrm{C}-\mathrm{O}$, and $\mathrm{C}-\mathrm{OH}$, respectively, similar to the previous literature. ${ }^{26}$ When $\mathrm{GO}$ is functionalized with AEPZ, the new peaks at 2930, 1455 and $1510 \mathrm{~cm}^{-1}$ appear, which are ascribed to the stretching vibration of $-\mathrm{CH}_{2}-$, the $\mathrm{C}-\mathrm{N}$ and $\mathrm{N}-\mathrm{H}$ groups in AEPZ, respectively. It obviously shows that the intensity of the $\mathrm{C}=\mathrm{O}$ peak decreases greatly for the reduction of GO. The results indicate that AEPZ has been successfully grafted onto the surface of GO. As can be seen from the spectrum of FGO, the distinctive absorptions appear: the peaks at 1256,1050 and $880 \mathrm{~cm}^{-1}$ are assigned to the symmetric and asymmetric stretching vibration of $\mathrm{P}=0$; the peak at $1311 \mathrm{~cm}^{-1}$ is ascribed to the deformation vibration of P-C; the peak at $1455 \mathrm{~cm}^{-1}$ is assigned to the $\mathrm{C}-\mathrm{N}$ group; the peaks at 1640,1730 and $2930 \mathrm{~cm}^{-1}$ are assigned to the stretching of $\mathrm{C}=\mathrm{C}, \mathrm{C}=\mathrm{O}$ and $-\mathrm{CH}_{2}-$ groups, respectively. ${ }^{19-21}$ These results clearly suggest that the hyper-branched flame retardant is successfully bonded to $\mathrm{GO}$.

The XRD patterns of GO, AEPZ-GO and FGO are presented in Figure $2 b$. The XRD spectrum of GO exhibits a sharp diffraction peak at $10.3^{\circ}$, whereas the intensity of this peaksignificantly decreasesfromAEPZ-GO to FGO, indicating that $\mathrm{GO}$ is chemically reduced by AEPZ and hyper-branched flame retardant grafted on the surface of GO disordered the stacking structure of GO sheets. The high molecular weight of the hyper-branched flame retardant exfoliates the GO sheets and restrains their reaggregation. Therefore, functionalized GO sheets with the hyperbranched flame retardant not only make their exfoliation into individual sheets available but also facilitate their 
uniform dispersion in PE matrix.

XPS is employed to clarify the chemical components on the surface of GO and FGO. The $\mathrm{C}_{1 \mathrm{~s}}, \mathrm{~N}_{1 \mathrm{~s}}$ and total spectra obtained from XPS are displayed in Figure 3. The results clearly show that the new peaks at 134.7, 190.9 and $400 \mathrm{eV}$ appear in

the XPS spectra of FGO, which are attributed to the $P_{2 p}, P_{2 s}$ and $N_{1 s}$ of the hyper-branched flame retardant. Figure $3 b$ gives the C1s XPS spectra of FGO with peak-fitting curves. The peaks at 284, 286.3 and $288.1 \mathrm{eV}$ are assigned to the carbon atoms in $\mathrm{C}=\mathrm{C} / \mathrm{C}-\mathrm{C}, \mathrm{C}-\mathrm{O}$ and $\mathrm{C}=\mathrm{O}$, respectively. Besides, an additional component at $285.4 \mathrm{eV}$ is observed from FGO, which originates from the formation of a $\mathrm{C}-\mathrm{N}$ bond via the surface modification of GO. As shown in Figure $3 c$, the $\mathrm{N}_{1 \mathrm{~s}}$ XPS spectra of FGO demonstrate that the hyper-branched flame retardant is grafted on the surface of $\mathrm{GO}$, because the peaks at 399.3 and $402 \mathrm{eV}$ are assigned to the $\mathrm{N}-\mathrm{C}$ and $\mathrm{N}-\mathrm{H}$, respectively. These results suggest that $\mathrm{GO}$ is successfully functionalized by the hyper-branched flame retardant using the amidation reaction and Michael addition reaction.

TEM and SEM are further used to investigate the morphology and microstructure of GO and FGO. As shown in Figure $4 \mathrm{a}, \mathrm{GO}$ sheets exhibit a wrinkled and folded structure with the diameter of several micrometers. Compared to $\mathrm{GO}$, the surface of FGO appears to be rough and clearly covered by the addends, which are attributed to the grafting of flame retardant (Figure $4 b, c)$. Furthermore, as shown in Figure 4d, SEM morphology of FGO shows an isotropic structure and very desultory stacking, suggesting little aggregations. The results show that the functionalized GO enhances the electrostatic repulsion of the layer of graphene, and then reduces the reaggregation of graphene sheet.

Thermal properties of GO and FGO in nitrogen atmosphere are investigated by TGA, and the related results are shown in Figure 5 and Table 1. As shown in Figure 5, the initial mass loss in stage of GO below $100{ }^{\circ} \mathrm{C}$ is ascribed to the evaporation of the adsorbed water, and the maximum mass loss temperature $\left(T_{\text {max }}\right)$ around $200{ }^{\circ} \mathrm{C}$ is attributed to the decomposition of labile oxygen functional groups. For FGO, the $5 \%$ mass loss temperature $\left(T_{-5 \%}\right)$ increases from $112{ }^{\circ} \mathrm{C}(\mathrm{GO})$ to $179{ }^{\circ} \mathrm{C}$, suggesting that the labile oxygen groups in $\mathrm{GO}$ are removed after the reaction with the hyper-branched flame retardant. The $T_{\max }$ of FGO increases to $229^{\circ} \mathrm{C}$ compared to that of GO, which is attributed to that the functionalization of GO with the hyper-branched flame retardant improves the thermal stability of FGO.

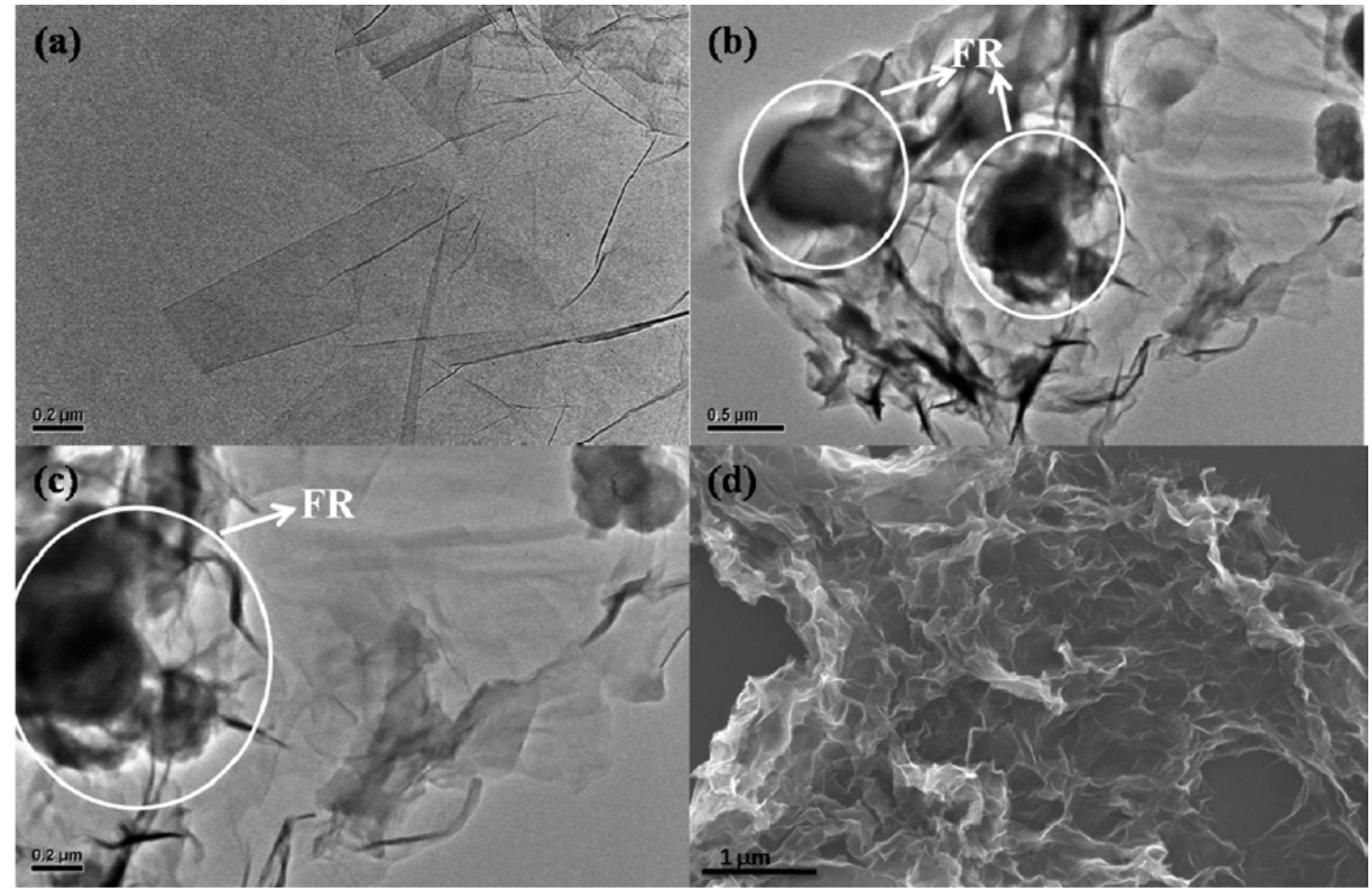

Figure 4. TEM photographs of GO (a), FGO (b,c) and SEM photographs of FGO (d). 


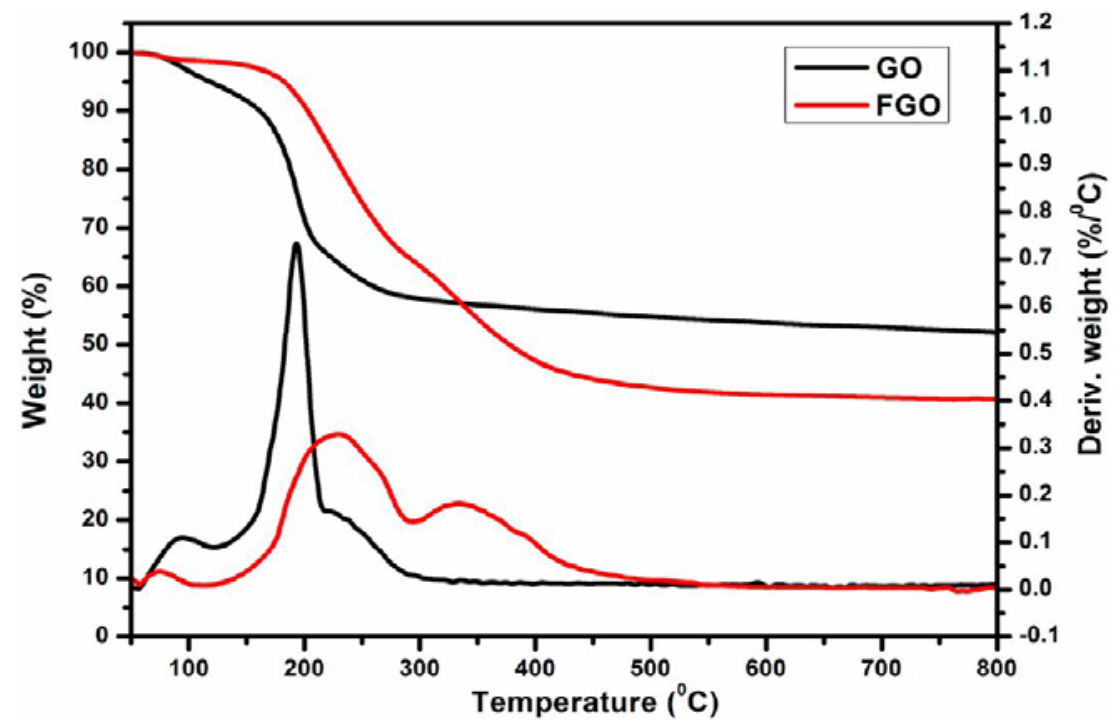

Figure 5. TGA and DTG curves of GO and FGO in nitrogen.

Table 1. Relative Data of TGA and DTG for GO and FGO in Nitrogen

\begin{tabular}{cccc} 
& \multicolumn{2}{c}{$\begin{array}{r}\text { temperature at specific } \\
\text { weight loss }\end{array}$} & \\
\cline { 2 - 3 } sample & $T-5 \%\left({ }^{\circ} \mathrm{C}\right)$ & $T_{\max }$ & residues at $800{ }^{\circ} \mathrm{C} \mathrm{( \% )}$ \\
GO & 112.4 & 193.3 & 52.1 \\
FGO & 179.1 & 229.1 & 40.7 \\
\hline
\end{tabular}

Morphology and Gel Content of XLPE-Based Nano- composites. The morphology of the XLPE nanocomposites with the nanofiller loading of $1 \mathrm{wt} \%$ is evaluated by TEM technique, as shown in Figure 6. A homogeneous and uniform dispersion of FGO nanosheets is observed in XLPE-FGO nanocomposites. However, in the case of XLPE-GO nanocomposites, the reaggregations and heterogeneous dispersion of the nanofillers are visible. The agglomeration of GO nanosheets in XLPE matrix results from the poor compatibility of the oxygenated group on the surface of GO with the nonpolar polymer matrix. On the contrary, the good dispersion of FGO in the XLPE matrix due to that the vinyl functional groups in FGO are chemically bonded into the cross-linked networks together with matrix.

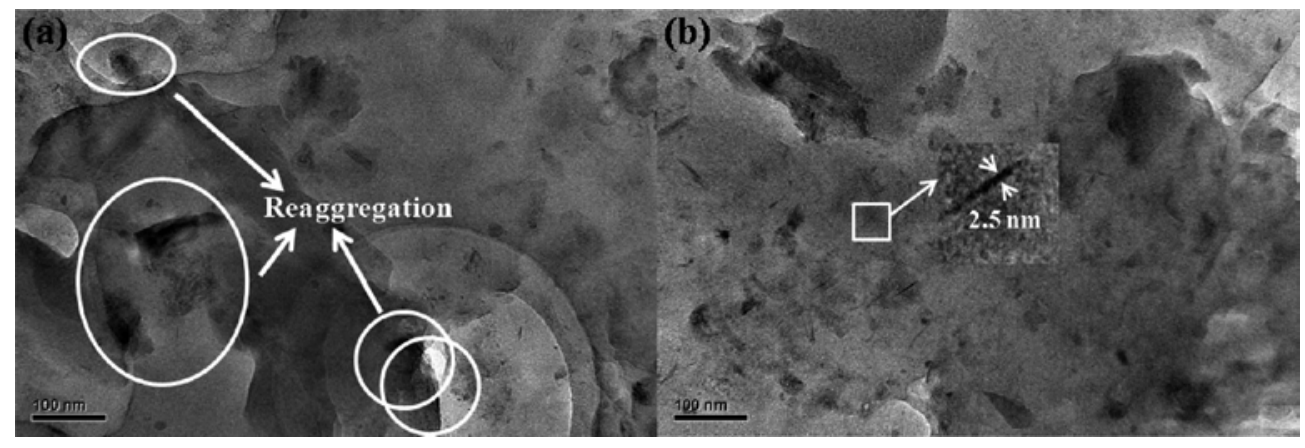

Figure 6. TEM photographs of dispersion of GO (a) and FGO (b) in XLPE matrix.

Figure 7 shows the gel contents of XLPE, XLPE-GO and XLPE-FGO nanocomposites. At the irradiation dose of 140 KGy, the gel content of virgin XLPE is up to the $85 \%$, suggesting that XLPE possess the excellent mechanical properties and antithermal ageing properties. In the case of the XLPE-GO nanocomposites, the gel content declines slightly with the amount of GO increasing, which may attribute to the hydroxyl and carboxyl groups on the surface of GO to effect the matrix cross-linked. However, the gel content increases via the FGO added, due to the vinyl group on the surface of FGO promotes the matrix cross-linked. 


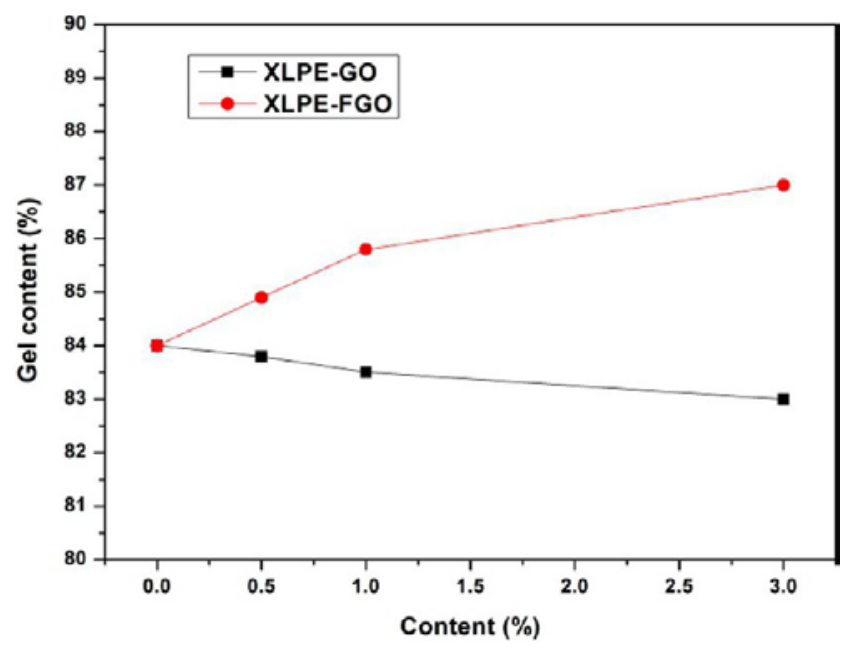

Figure 7. Gel contents of XLPE, XLPE-GO and XLPE-FGO nanocomposites.

Flame Retardancy. A cone calorimeter is a useful bench- scale tool to evaluate the flammability behaviors of polymer composites. LOI tests are widely employed to assess the efficacy of fire performance. Figure 8 gives the heat release rate (HRR) and total heat release (THR) versus time curves of the XLPE nanocomposites, and some related parameters recorded from the cone calorimeter tests, such as the time to ignition (TTI), the time to peek heat release rate, the peak heat release rate (PHRR) the THR and the LOI are also compiled in Table 2. From Figure 8, it can be observed that pure XLPE burns very rapidly after ignition, with the PHRR and THR values of $1741 \mathrm{~kW} / \mathrm{m}^{2}$ and 103.1 $\mathrm{MJ} / \mathrm{m}^{2}$, respectively. As expected, incorporating $0.5 \% \mathrm{GO}$ into XLPE turns the peak heat release rate to decrease to $1671 \mathrm{~kW} / \mathrm{m}^{2}$. Furthermore, with the content of GO increasing to $1 \%$ and $3 \%$, the PHRR values of the XLPE composites are reduced by $8.3 \%$ and $9.0 \%$, respectively. The results show that the addition of GO is not very effective in the reduction of PHRR, due to the balance of physical barrier and heat conduction effects of the GO. ${ }^{27}$ However, in the case of XLPE-FGO nanocomposites, the PHRR decreases obviously, compared to the pure XLPE. The PHRR value is reduced by $10.2 \%, 27.9 \%$ and $28.9 \%$ for XLPE-FGO $-0.5,-1.0$ and -3.0 , respectively, indicating that FGO is more effective in enhancing the flame retardancy of polymers. The TTI of XLPE-FGO is predated with increasing the FGO additive, attributed to the decomposition of flame retardant on the surface of FGO. The results come to the conclusion that the functionalized GO with hyper-branched flame retardant promote the char formation of GO, enhance the physical barrier effect and decrease the heat conduction effect of GO.

The incorporation of GO slightly improves the THR of the XLPE composites, due to the excellent heat conduction of GO. Moreover, the THR value slightly decreases with the increase in the amount of GO. However, for the sample of XLPE-FGO, the THR value decreases distinctly as the amount added of FGO increases, suggesting that the functionalized GO enhance the flame resistance of the composites. Fire growth index (FGI) is defined as the proportion of PHRR and time to PHRR, which is usually used to evaluate the fire safety of materials. The lower FGI value means less hazards caused in fire condition. As the amount of GO increases, the FGI value decreases.

As shown in Table 2, the LOI value of pure XLPE is only 18.5\%, but the LOI value goes up after GO and FGO incorporated to theXLPE. Meanwhile, the LOI of XLPE-FGO is slightly higher than the XLPE-GO sample in the same additive. The LOI test is the measurement based on the self- extinguishing property. Although the nanocomposites burn slowly after igniting, they cannot self-extinguish until most of the fuel combusts. Thus, XLPE-FGO nanocomposites show little effect on improving the LOI value, but they also show the best flame retardant efficiency than the others. 

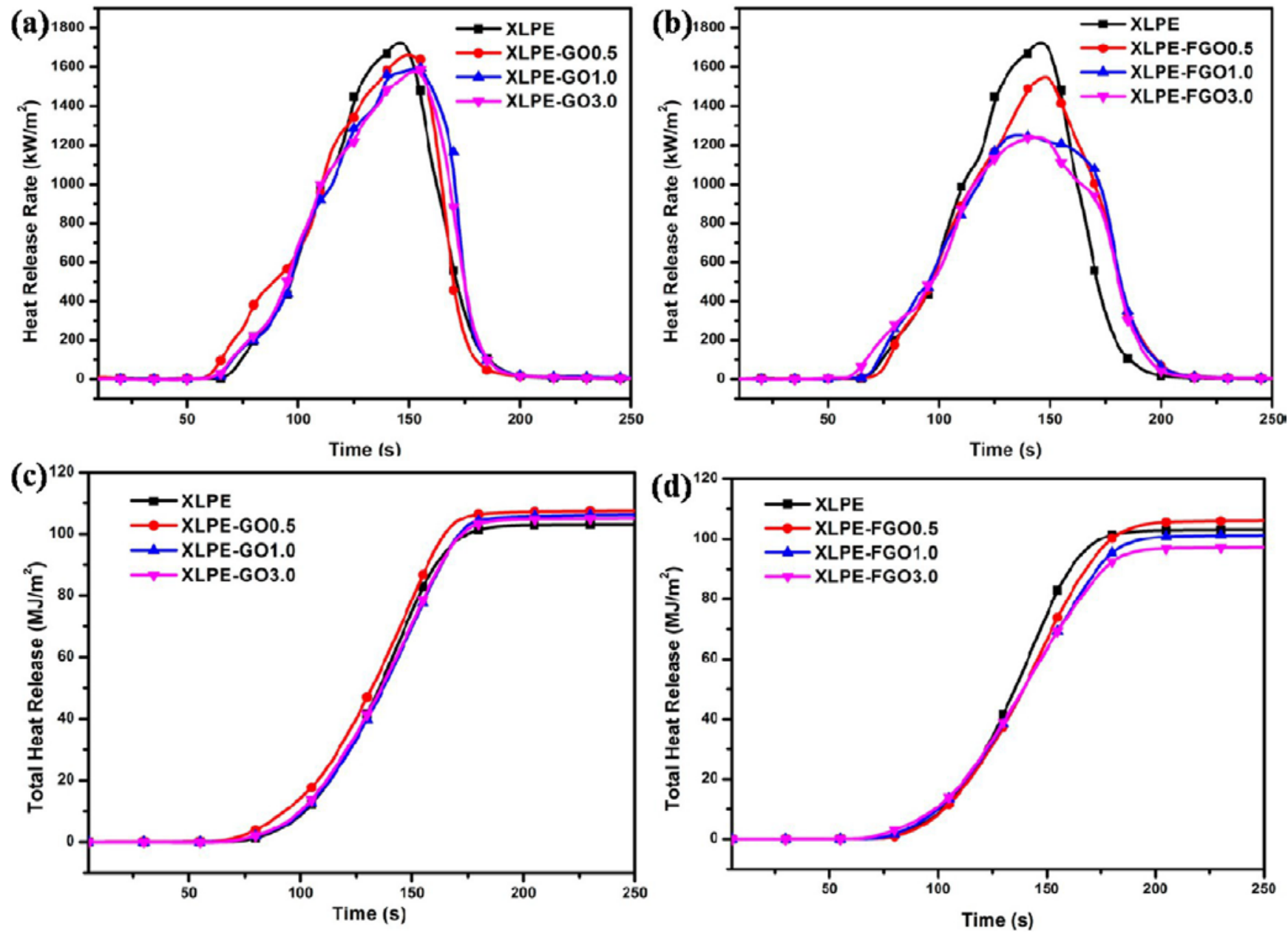

Figure 8. HRR and THR curves of XLPE, XLPE-GO and FGO nanocomposites.

\begin{tabular}{|c|c|c|c|c|c|c|}
\hline sample & TTI (s) & time to PHRR (s) & PHRR $\left(\mathrm{KW} / \mathrm{m}^{2}\right)$ & THR $\left(\mathrm{MJ} / \mathrm{m}^{2}\right)$ & $\mathrm{FGI}\left(\mathrm{kW} / \mathrm{m}^{2} \mathrm{~s}\right)$ & LOI (\%) \\
\hline XLPE & 66 & 150 & 1741.3 & 103.1 & 11.61 & 18.5 \\
\hline XLPE-GO -0.5 & 51 & 150 & 1671.4 & 107.8 & 11.14 & 19.0 \\
\hline XLPE-GO -1.0 & 55 & 155 & 1595.8 & 106.5 & 10.29 & 19.0 \\
\hline XLPE-GO -3.0 & 65 & 155 & 1585.6 & 105.1 & 10.22 & 19.5 \\
\hline XLPE-HGO -0.5 & 66 & 150 & 1563 & 106.1 & 10.42 & 19.0 \\
\hline XLPE-HGO -1.0 & 62 & 135 & 1254 & 101.3 & 9.29 & 19.5 \\
\hline XLPE-HGO -3.0 & 52 & 145 & 1241 & 97.3 & 8.56 & 20.5 \\
\hline
\end{tabular}

Thermogravimetric Analysis. The thermal stability of XLPE-GO and XLPE-FGO nanocomposites is evaluated by TGA under nitrogen, and the TGA curves are displayed in Figure 9. The onset decomposition temperature $\left(T_{\mathrm{d}}\right)$ can be defined as the temperature at which the mass loss is $5 \%$, which is used to evaluate the relative thermal stability of the composites. The parameters obtained from TGA curves, including $T_{\mathrm{d}}$, the temperature of maximum rate of weight loss $\left(T_{\max }\right)$ and the char yield at $700{ }^{\circ} \mathrm{C}$, are listed in Table 3.

The $T_{\mathrm{d}}$ of pure XLPE is $436.1^{\circ} \mathrm{C}$, and its TGA curve shows one single mass-loss stage, which is assigned to the decomposition of the macromolecular chains. The range of decomposition stage is from 390 to $530{ }^{\circ} \mathrm{C}$ with a single DTG peak at $484.5^{\circ} \mathrm{C}\left(T_{\max }\right)$, and the char yield at $700{ }^{\circ} \mathrm{C}$ is about $0.8 \%$. In the case of XLPE-GO nanocomposites, the value of $T_{\mathrm{d}}, T_{\max }$ and char residue percentage of XLPE nanocomposites increase gradually, when the amount of GO increases from $0.5 \%$ to $3 \%$. The thermal stability of the nanocomposites is improved after incorporation of GO, due to the heat and mass barrier effects of GO. In the case of XLPE-FGO nanocomposites, the similar behaviors of XLPE-GO nanocomposites are also observed with the amount of FGO increasing. However, the interesting phenomenon is that the values of $T_{-5 \%}, T_{\max }$ and char residue are improved compared to those of the XLPE-GO nanocomposites, with the equivalent loading of nanofiller, indicating that the well dispersion and the cross- linked structure strengthen the barrier effect of GO. Therefore, it can be deduced that the functionalization of GO is beneficial to enhance the thermal stability of polymer matrix. 

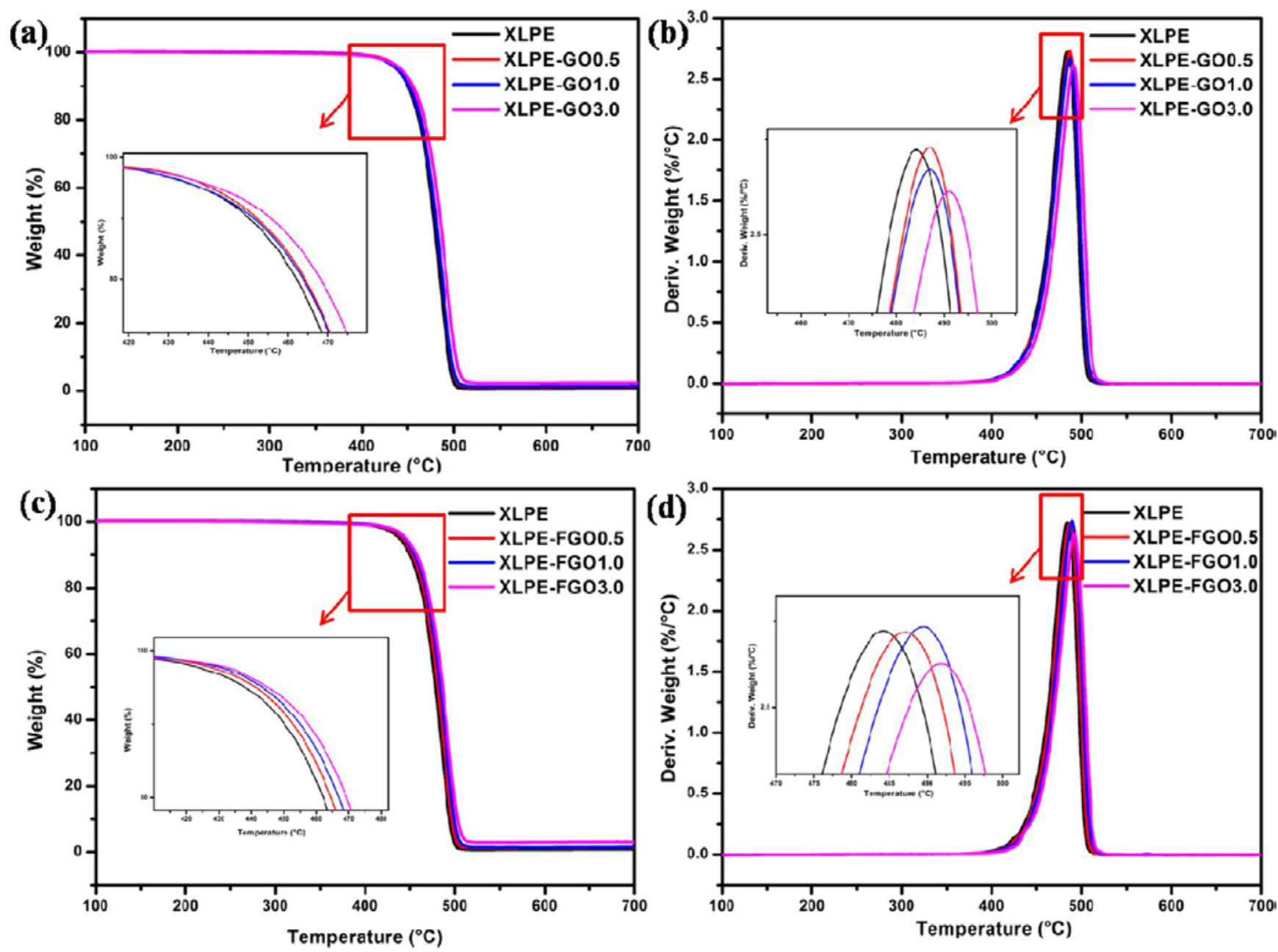

Figure 9. TGA and DTG curves of XLPE, XLPE-GO and FGO nanocomposites in nitrogen.

Table 3. TGA Data of XLPE Nanocomposites in Nitrogen

\begin{tabular}{lccc} 
& \multicolumn{2}{c}{ temperature at specific } \\
& \multicolumn{2}{c}{ weight loss $\left({ }^{\circ} \mathrm{C}\right)$} & \\
\cline { 2 - 3 } & $T_{\mathrm{d}}\left({ }^{\circ} \mathrm{C}\right)$ & $T_{\max }\left({ }^{\circ} \mathrm{C}\right)$ & residues at $700{ }^{\circ} \mathrm{C}(\%)$ \\
sample & 436.1 & 484.5 & 0.8 \\
XLPE & 438.8 & 486.8 & 1.3 \\
XLPE-GO -0.5 & 439.3 & 487.0 & 1.5 \\
XLPE-GO -1.0 & 439.8 & 491.0 & 2.4 \\
XLPE-GO -3.0 & 439.4 & 487.1 & 1.5 \\
XLPE-FGO -0.5 & 441.3 & 489.5 & 1.6 \\
XLPE-FGO -1.0 & 443.2 & 491.9 & 3.2 \\
XLPE-FGO -3.0 & 44.2 &
\end{tabular}

Oxidative Induction Temperature and Oxidative Induction Time. The OITm and OITs measurements conducted by DSC are used to evaluate the antioxidant behavior of XLPE nanocomposites on dynamic and steady temperature. The DSC curves and the OITm and OITs results of XLPE and its nanocomposites are shown in Figure 10.

As compared to the pure XLPE, the OITm value decreases for the XLPE-GO composites. It is reported that the oxygen molecules could be absorbed on the surface of graphene like the fullerenes and carbon nanotube. ${ }^{28,29}$ These oxygen molecules interact with the hydroxyl and carboxyl groups on the surface of GO, which induce the formation of some active radicals. Such radicals serving as the catalyst to accelerate the reaction of thermal oxidation leads to the reduction in the

OITm value. On the contrary, the addition of FGO into the XLPE matrix exhibits a positive effect on the OITm value. The carboxyl groups on the surface of $\mathrm{GO}$ are reacted after the surface modification by the hyper-branched flame retardant, resulting in the reduction of the radicals formed. Moreover, the structure of the cross-linked network between FGO and XLPE may also hinder the shift of free radicals in the thermal oxidative degradation. 
The results of OITs show that the XLPE-GO and XLPE-FGO exhibit longer antioxidative times than that of virgin XLPE. It is different with the OITm results, because the measuring OITm is in a rapid heating process condition, which cannot lead to the degradation of the hydroxyl and carboxyl groups on the surface of GO. As in the OITs measurement, the temperature is sustained for a relative long time, decomposing the hydroxyl and carboxyl groups of $\mathrm{GO}$, and maintaining the capability of graphene to capture oxygen. In the case of the XLPE-FGO, because of the higher thermal stability and the capability of cross-linked with matrix of FGO to enhance the property of captive oxygen, the OITs of XLPE-FGO is longer than that of the XLPE-GO. Therefore, incorporating FGO into XLPE exhibits superior antioxidation property over GO.

Mechanical Properties and Thermal Ageing. As shown in Figure 11a, the tensile strength (TS) and elongation at break (EB) of XLPE nanocomposites are listed. It can be observed that the TS and EB of XLPE-GO increase compared to the XLPE, which is assigned to the excellent elastic modulus and intrinsic strength of graphene sheets. After functionalisation by a hyper-branched flame retardant, the good dispersion of FGO and cross-linked to matrix can promote further increases of the TS and EB. With the additive amount of the GO and FGO from $0.5 \%$ to $3 \%$, they also perform increased TS and EB.
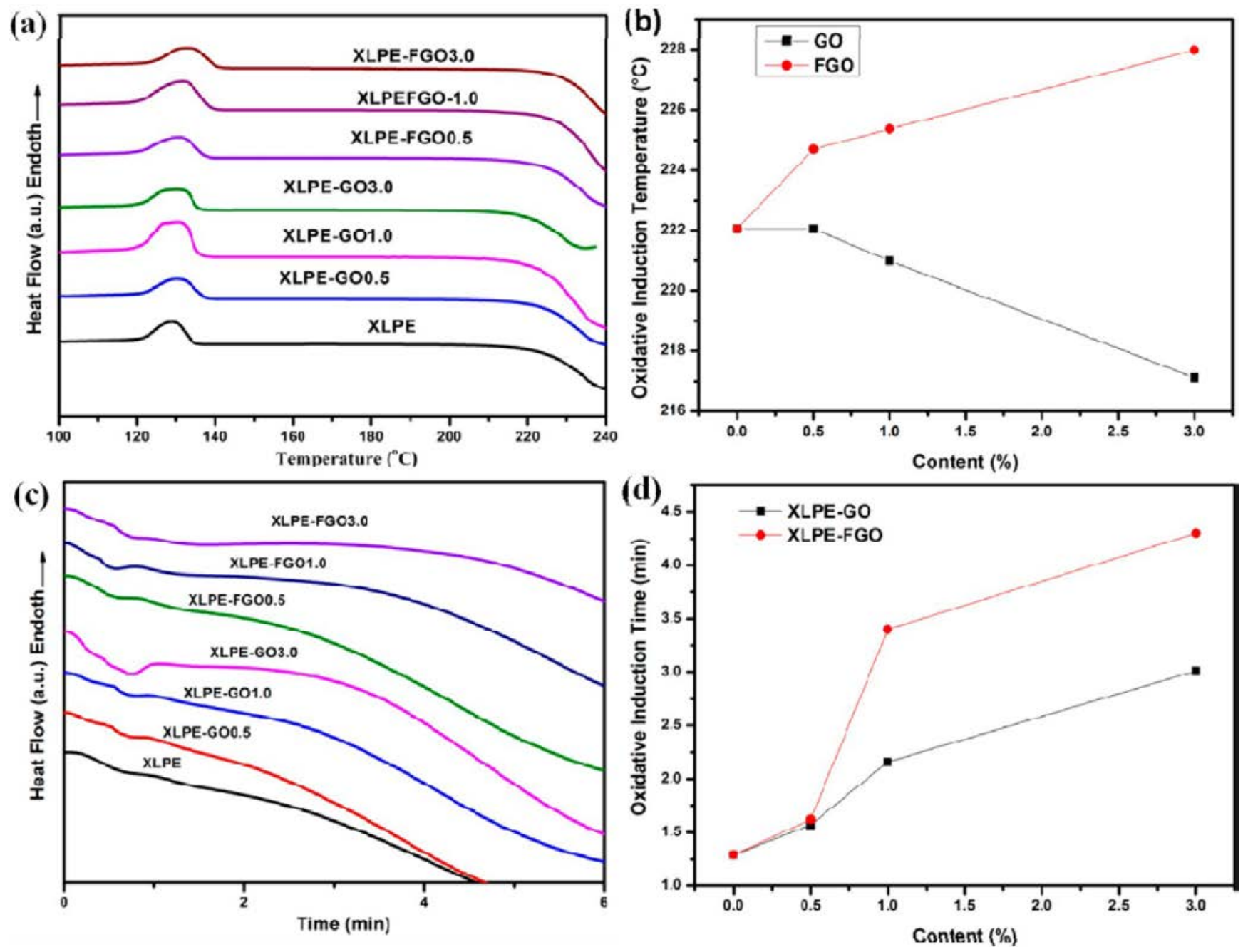

Figure 10. OITm curves (a) and the OITm data (b), OITs curves (c) and the OITs data (d) of XLPE, XLPE-GO and FGO nanocomposites. 
The thermal ageing test has been measured in the air oven at $135^{\circ} \mathrm{C}$ for 18 days. The TS and EB retention rates of XLPE and XLPE nanocomposites via the different thermal ageing time are displayed in Figure 11b,c. The results show that the pure XLPE is entirely cracked after ageing 3 days, whereas the TS retention maintains a declining tendency and the EB retention raises to a peak and then declines, because of the thermal creep deformation of the macromolecule. In the case of the XLPE-GO, TS and EB retention of XLPE-GO nanocomposites decrease quickly compared to the pure XLPE and XLPE-FGO over the initial ageing time. The hydroxyl and carboxyl groups of GO accelerate the materials' ageing, suggesting the results of OITm. However, XLPE-GO can maintain the mechanical properties after ageing for 3 days, whereas the XLPE cracked. As for the fully cracked, the ageing time of XLPE-FGO 3.0, sustaining the ageing 18 days, is the longest specimen of all, and the ageing time of all the XLPE-FGO samples are delayed compared to the pure XLPE and XLPE-GO. It is because they are cross-linked with the polymer and the thermal stability of FGO has a significant effect on the thermal ageing progress of XLPE nanocomposites. The above results are in accordance with the results of OITs.

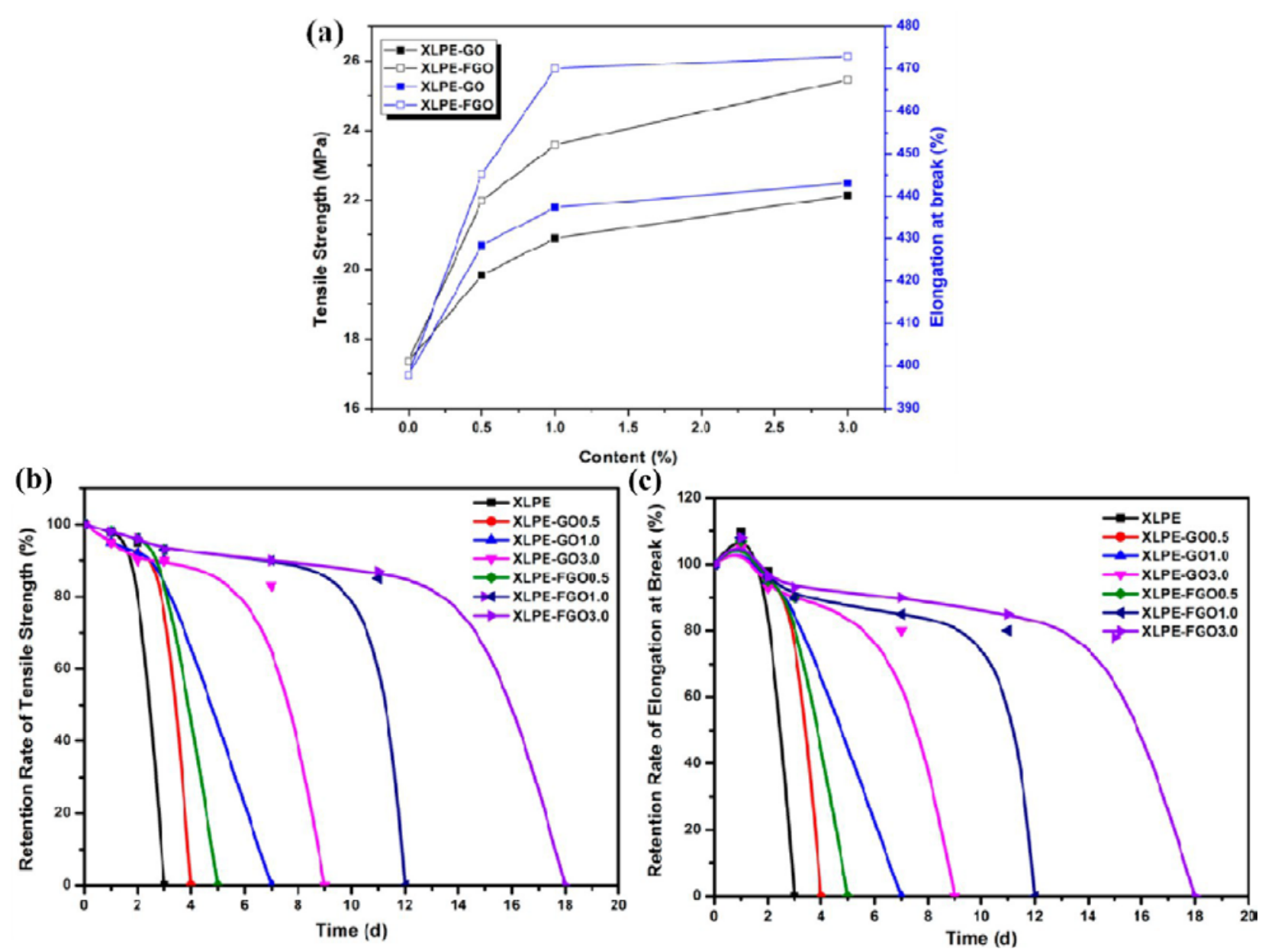

Figure 11. The relative data of mechanic properties (a) and retention rate of TS (b) and EB (c) in ageing test of XLPE, XLPE-GO and XLPE-FGO nanocomposites. 


\section{CONCLUSION}

In this paper, functionalized GO with a hyper-branched flame retardant was designed and synthesized and then applied to prepare a series of XLPE-based nanocomposites. The results of FTIR, XRD, XPS, SEM and TEM indicated that the hyper-branched flame retardant was successful synthesized and covalentlygrafted onto the surface of GO.In addition, the TGA results showed that thermal stability of GO was improved after the functionalization. The incorporation of FGO into XLPE matrix obviously improved the dispersion of graphene nano- sheets compared to the XLPE-GO nanocomposites. The fire safety index of XLPE-FGO nanocomposites, such as PHRR, THR, FGI and LOI, were superior over those of XLPE-GO nanocomposites, due to the better dispersion of nanofillers in matrix and the crosslinked structure of nanocomposites. Moreover, the XLPE-FGO nanocomposites had higher char yields, better thermal stability and enhanced antioxidation properties compared to the XLPE-GO nanocomposites, which was believed to be that the surface modification of GO reinforced the barrier effect and reduced the formation of radicals during thermal degradation process. Meanwhile, the XLPE-FGO had improved the mechanical properties and significantly delayed the ageing time in a thermal ageing test. Functionalized GO with hyper-branched flame retardant presented herein will provide an effective strategy to improve the thermal stability and fire safety properties in nonpolar polymers.

\section{AUTHOR INFORMATION}

Corresponding Authors

*J. Wang. Fax/Tel: +86-551-63606463. E-mail: wangj@ustc. edu.cn.

*Y. Hu. Fax/Tel: +86-551-63601664. E-mail: yuanhu@ustc. edu.cn.

Notes

The authors declare no competing financial interest.

\section{ACKNOWLEDGMENTS}

The work was financially supported by the National Basic Research Program of China (973 Program) (2012CB719701), National Natural Science Foundation of China (No. 51303167), and UCLan Biomedical Technology (Shenzhen) LTD. for the project 'The Application of Nanoscience to Fire Safety'.

\section{REFERENCES}

(1) Novoselov, K. S.; Geim, A. K.; Morozov, S. V.; Jiang, D.; Zhang, Y.; Dubonos, S.V.; Grigorieva, I.V.; Firsov, A. A. Electricfield effect in atomically thin carbon films. Science 2004, 306, 666-669.

(2) Pang, H.; Chen, C.; Zhang, Y. C.; Ren, P. G.; Yan, D. X.; Li, Z. M. The effect of electric field, annealing temperature and filler loading on the percolation threshold of polystyrene containing carbon nanotubes and graphene nanosheets. Carbon 2011, 49, 1980-1988.

(3) Fang, M.; Wang, K. G.; Lu, H. B.; Yang, Y. L.; Nutt, S. Covalent polymer functionalization of graphene nanosheets and mechanical properties of composites. J. Mater. Chem. 2009, 19, 7098-7105.

(4) Salavagione, H. J.; Gomez, M. A.; Martınez, G. Polymeric modification of graphene through esterification of graphite oxide and Poly(vinyl alcohol). Macromolecules 2009, 42, 6331-6334.

(5) Schniepp, H. C.; Li, J. L.; McAllister, M. J.; Sai, H.; Herrera- Alonso, M.; Adamson, D. H. Functionalized single graphene sheets derived from splitting graphite oxide. J Phys. Chem. B 2006, 110, 8535-8539.

(6) McAllister, M. J.; Li, J. L.; Adamson, D. H.; Schniepp, H. C.; Abdala, A. A.; Liu, J. Single sheet functionalized graphene by oxidation and thermal expansion of graphite. Chem. Mater. 2007, 19, 4396-4403.

(7) Berger, C.; Song, Z.; Li, X.; Wu, X.; Brown, N.; Naud, C. Electronic confinement and coherence in patterned epitaxial graphene. Science 2006, 312, 1191-1196.

(8) Stankovich, S.; Dikin, D. A.; Piner, R. D.; Kohlhaas, K. A.; Kleinhammes, A.; Jia, Y. Synthesis of graphene-based nanosheets via chemical reduction of exfoliated graphite oxide. Carbon 2007, 45, 1558-1565.

(9) Hyunwoo, K.; Ahmed, A. A.; Christopher, W. M. Graphene/ Polymer Nanocomposites. Macromolecules 2010, 43, 6515-6530.

(10) Loh, K. P.; Bao, Q. L.; Ang, P. K.; Yang, J. X. The chemistry of graphene. J. Mater. Chem. 2010, 20, 2277-2289.

(11) Lin, Y.; Jin, J.; Song, M. Preparation and characterisation of covalentpolymerfunctionalizedgrapheneoxide.J. Mater.Chem. 2011, 21, 3455-3461.

(12) Huang, Y. J.; Qin, Y. W.; Zhou, Y.; Niu, H.; Yu, Z. Z.; Dong, J. Y. Polypropylene/Graphene Oxide Nanocomposites Prepared by In SituZiegler-NattaPolymerization. Chem. Mater. 2010,22,4096-4102.

(13) Stankovich, S.; Piner, R. D.; Nguyen, S. B. T.; Ruoff, R. S. Synthesis and exfoliation of isocyanate-treated graphene oxide nanoplatelets. Carbon 2006, 44, 3342-3347.

(14) Bon, S. B.; Valentini, L.; Verdejo, R.; Fierro, J. L.G; Peponi, L.; Lopez-Manchado, M. A.; Kenny, J. M. Plasma fluorination of chemically derived graphene sheets and subsequent modification with butylamine. Chem. Mater. 2009, 21, 3433-3438. 
(15) Chu, J.; Li, X.; Xu, P. Fluorescent features of CdTe nanorods grafted to graphene oxide through an amidation process. J. Mater. Chem. 2011, 21, 11283-11287.

(16) Lomeda, J. R.; Doyle, C. D.; Kosynkin, D. V.; Hwang, W. F.; Tour, J. M. Diazonium functionalization of surfactant-wrapped chemically converted graphene sheets. J. Am. Chem. Soc. 2008, 130, 16201-16206.

(17) Wang, X.; Xing, W. Y.; Zhang, P.; Song, L.; Yang, H. Y.; Hu, Y. Covalent functionalization of graphene with organosilane and its use as a reinforcement in epoxy composites. Compos. Sci. Technol. 2012, 72, 737-743.

(18) Chen, Z. X.; Lu, H. B. Constructing sacrificial bonds and hidden lengths for ductile graphene/polyurethane elastomers with improved strength and toughness. J. Mater. Chem. 2012, 22, 12479-12490.

(19) Bao, C. L.; Guo, Y. Q.; Song, L.; Kan, Y. C.; Qian, X. D.; Hu, Y. In situ preparation of functionalized graphene oxide/epoxy nanocomposites with effective reinforcements. J. Mater. Chem. 2011, 21, 13290-13298.

(20) Bao, C. L.; Guo, Y. Q.; Yuan, B. H.; Hu, Y.; Song, L. Functionalized graphene oxide for fire safety applications of polymers: a combination of condensed phase flame retardant strategies. J. Mater. Chem. 2012, 22, 23057-23063.

(21) Huang, G. B.; Chen, S. Q.; Tang, S. W.; Gao, J. R. A novel intumescent flame retardant-functionalized graphene: Nanocomposite synthesis, characterization, and flammability properties. Mater. Chem. Phys. 2012, 135, 938-947.

(22) Voit, B. I.; Lederer, A. Hyperbranched and highly branched polymer architectures: Synthetic strategies and major characterization aspects. Chem. Rev. 2009, 109, 5924-5973.

(23) Zhuo, D. X.; Gu, A. J.; Liang, G.Z.; Hu, J.T.; Yuan, L.; Chen, X. X. Flame retardancy materials based on a novel fully end-capped hyperbranched polysiloxaneand bismaleimide/diallylbisphenolAresin with simultaneously improved integrated performance. J. Mater. Chem. 2011, 21, 6584-6594.

(24) You, Y.Z.; Yan, J. J.; Yu, Z. Q.; Cui, M. M.; Hong, C. Y.; Qu, B. J. Multi-responsive carbon nanotube gel prepared via ultrasound- induced assembly. J. Mater. Chem. 2009, 19, 7656-7660.

(25) Wang, H. L.; Xu, S. P.; Shi, W. F. Photopolymerization behaviors of hyperbranched polyphosphonate acrylate and properties of the UV cured film. Prog. Org. Coat. 2009, 65, 417-424.

(26) Mo, Z. L.; Gou, H.; He, J. X.; Yang, P. P.; Feng, C.; Guo, R. B. Controllable synthesis of functional nanocomposites: covalently functionalize graphene sheets with biocompatible L-lysine. Appl. Surf. Sci. 2012, 258, 8623-8628.

(27) Bao, C. L.; Song, L.; Xing, W. Y.; Yuan, B. H.; Wilkie, C. A.; Huang, J. L.; Guo, Y. Q.; Hu, Y. Preparation of graphene by pressurized oxidation and multiplex reduction and its polymer nanocomposites bymasterbatch-basedmeltblending.J. Mater. Chem. 2012, 22, 6088-6096.

(28) Gorrasi, G.; Sorrentino, A. Photo-oxidative stabilization of carbon nanotubes on polylactic acid. Polym. Degrad. Stab. 2013, 98, 963-971.

(29) Eklund, P. C.; Rao, A. M.; Zhou, P.; Wang, Y.; Holden, J. M. Photochemical transformation of $\mathrm{C}_{60}$ and $\mathrm{C}_{70}$ films. Thin Solid Films $1995,257,185-203$. 